Case Report

\title{
Severe Left Ventricular Hypertrophy, Small Pericardial Effusion, and Diffuse Late Gadolinium Enhancement by Cardiac Magnetic Resonance Suspecting Cardiac Amyloidosis: Endomyocardial Biopsy Reveals an Unexpected Diagnosis
}

\author{
Nina P. Hofmann, ${ }^{1}$ Sorin Giusca, ${ }^{1}$ Karin Klingel, ${ }^{2}$ \\ Peter Nunninger, ${ }^{3}$ and Grigorios Korosoglou ${ }^{1}$ \\ ${ }^{1}$ Department of Cardiology \& Vascular Medicine, GRN Hospital Weinheim, 69469 Weinheim, Germany \\ ${ }^{2}$ Department of Molecular Pathology, University Hospital Tübingen, Tübingen, Germany \\ ${ }^{3}$ Department of Radiology, GRN Hospital Weinheim, 69469 Weinheim, Germany \\ Correspondence should be addressed to Grigorios Korosoglou; grigorios.korosoglou@grn.de
}

Received 31 January 2016; Revised 16 April 2016; Accepted 24 April 2016

Academic Editor: Gianluca Di Bella

Copyright ( 2016 Nina P. Hofmann et al. This is an open access article distributed under the Creative Commons Attribution License, which permits unrestricted use, distribution, and reproduction in any medium, provided the original work is properly cited.

\begin{abstract}
Left ventricular (LV) hypertrophy can be related to a multitude of cardiac disorders, such as hypertrophic cardiomyopathy (HCM), cardiac amyloidosis, and hypertensive heart disease. Although the presence of LV hypertrophy is generally associated with poorer cardiac outcomes, the early differentiation between these pathologies is crucial due to the presence of specific treatment options. The diagnostic process with LV hypertrophy requires the integration of clinical evaluation, electrocardiography (ECG), echocardiography, biochemical markers, and if required CMR and endomyocardial biopsy in order to reach the correct diagnosis. Here, we present a case of a patient with severe LV hypertrophy (septal wall thickness of $23 \mathrm{~mm}, \mathrm{LV}$ mass of $264 \mathrm{~g}$, and LV mass index of $147 \mathrm{~g} / \mathrm{m}^{2}$ ), severely impaired longitudinal function, and preserved radial contractility (ejection fraction $=55 \%$ ), accompanied by small pericardial effusion and diffuse late gadolinium enhancement (LGE) by cardiac magnetic resonance (CMR). Due to the imaging findings, an infiltrative cardiomyopathy, such as cardiac amyloidosis, was suspected. However, amyloid accumulation was excluded by endomyocardial biopsy, which revealed the presence of diffuse myocardial fibrosis in an advanced hypertensive heart disease.
\end{abstract}

\section{Introduction}

Increased left ventricular (LV) wall thickness, referred to as LV hypertrophy, is a powerful predictor of cardiovascular morbidity and mortality [1]. LV hypertrophy can be related to a multitude of cardiac or systemic diseases such as hypertrophic cardiomyopathy (HCM), cardiac amyloidosis, Anderson-Fabry disease, and hypertensive heart disease [2-4]. The clinical presentation of all of these disorders is highly variable from being asymptomatic to sudden death, whereas they usually lead to congestive heart failure in their advanced stages.

Amyloidosis is characterized by the extracellular deposition of amyloid, as a result of misfolding of a precursor protein, the most common of which are light chains, transthyretin, and serum amyloid A [5]. Cardiac involvement is common and represents an adverse predictor of outcome with amyloidosis [4]. HCM, on the other hand, is caused by mutation of sarcomeric genes and is associated with concentric LV hypertrophy [3]. In some patients, HCM progresses to congestive heart failure with poor clinical outcome [6]. In addition, arterial hypertension is the most common cause of LV hypertrophy due to pressure overload, which can also progress to hypertensive heart disease associated with heart failure with or without preserved LV ejection fraction [2]. Due to advances in the antihypertensive treatment, the burden of hypertensive heart diseases decreased during the last decades [7]. 
TABLE 1: Serum and urine laboratory findings.

\begin{tabular}{lc}
\hline High-sensitive troponin T & $31.8 \mathrm{pg} / \mathrm{mL}$ \\
Serum creatinine & $1.28 \mathrm{mg} / \mathrm{dL}$ \\
eGFR (estimated glomerular filtration & $>60 \mathrm{~mL} / \mathrm{min} * 1.73 \mathrm{~m}^{2}$ \\
rate) & $0.8 \mathrm{mg} / \mathrm{L}$ \\
C-reactive protein (CRP) & $41 \mathrm{mg} / \mathrm{L}$ \\
Free light chains in urine & $<5.3 \mathrm{mg} / \mathrm{L}$ \\
Free Lambda light chains in urine & $97 \mathrm{mg} / \mathrm{L}$ \\
Proteins in urine & $195 \mathrm{mg} / 24 \mathrm{~h}$ \\
\hline
\end{tabular}

In clinical routine, the diagnostic workup of patients with LV hypertrophy is important due to specific therapeutic strategies aiming at the different disease etiologies. Here, we present a case where the presence of cardiac amyloidosis was suspected based on cardiac imaging findings. However, endomyocardial biopsy revealed an unexpected diagnosis.

\section{Case Presentation}

A 56-year-old male patient was referred to our department due to exertional dyspnea and gradual decrease of his exercise tolerance within the last 6 months. The patient had no history of cardiac diseases and no history of arterial hypertension, hyperlipidemia, and diabetes mellitus. Physical examination revealed normal heart sounds, no murmurs, and mild peripheral edema with clear lung fields. Blood pressure was elevated at $180 / 90 \mathrm{mmHg}$ with normal pulse of $58 \mathrm{bpm}$. An ECG suggested a sinus rhythm without signs of myocardial ischemia or low QRS voltage but negative T-waves in V4-V6. Echocardiography, on the other hand, revealed the presence of severe LV hypertrophy (Figures 1(a)$1(\mathrm{f})$ ) with septal wall thickness of $20 \mathrm{~mm}$, preserved radial wall motion, and LV ejection fraction but strongly diminished longitudinal function (Figures $1(\mathrm{~g})$ and $1(\mathrm{~h})$ ) (mitral annular plane systolic excursion (MAPSE) $=7 \mathrm{~mm}$; mean longitudinal strain $=-11.1 \%$; ejection fraction $=55 \%)$. In addition, granular sparkling of the myocardium was observed and small pericardial effusion was noticed, without hemodynamic relevance (orange arrows in Figures 1(b) and 1(e)). High-sensitive troponin T (hsTnT) was elevated $(32 \mathrm{pg} / \mathrm{mL})$, whereas renal function and C-reactive protein were normal. In addition, light chains and protein in urine were within the normal range (Table 1 ).

Because echocardiographic findings were indicative of a myocardial infiltrative disease, cardiac magnetic resonance (CMR) was subsequently performed. CMR confirmed the presence of severe LV hypertrophy (septal wall thickness: $23 \mathrm{~mm}$; lateral wall thickness: $18 \mathrm{~mm}$; LV mass: $264 \mathrm{~g}$; and LV mass index: $147 \mathrm{~g} / \mathrm{m}^{2}$ ), small pericardial effusion, and impaired longitudinal wall motion with an ejection fraction of 54\% (Figures 2(a)-2(f)). In addition, late gadolinium enhancement exhibited a diffuse LGE pattern in the 4chamber view and patchy LGE pattern in the inferiorlateral wall, suspecting cardiac amyloidosis (Figures 2(g) and $2(\mathrm{~h})$ ). Based on all the echocardiographic and CMR imaging findings and due to suspected cardiac amyloidosis, the patient was scheduled for cardiac catheterization and endomyocardial biopsy. Coronary angiography excluded the presence of significant coronary artery disease and several biopsies could be harvested from the left ventricle. From paraffin-embedded cardiac tissue, routine cardiopathologic staining and immunohistochemistry were performed. Congo red stain excluded the presence of amyloid in the heart. In addition, no apple-green birefringence was observed in the heart tissue of our patient under polarized light in Figures 3(a) and 3(b). Masson's trichrome stain, on the other hand, demonstrated the presence of cardiomyocyte hypertrophy and a diffuse interstitial fibrosis including microfoci of collagen scars (blue areas in Figure 3(c)). Quantification analysis as obtained by the programme Quantuepatho showed an area of fibrosis of $7 \%$ in our patient (Figure 3(d)) [8]. This percentage is higher compared to normal heart tissue, which contains very low amounts of fibrous perimysial collagen and less than 3\% fibrosis [9]. In addition, immunohistochemical stains for desmin and CD68 stains suggested the absence of myocardial texture disorders and macrophage infiltration (Figures 3(a)-3(f)).

The patient was put on antihypertensive medication with $190 \mathrm{mg}$ metoprolol, $10 \mathrm{mg}$ ramipril, $250 \mathrm{mg}$ clonidine retard, $50 \mathrm{mg}$ dihydralazine, $10 \mathrm{mg}$ amlodipine, $8 \mathrm{mg}$ doxazosin, and $50 \mathrm{mg}$ triamterene with $25 \mathrm{mg}$ hydrochlorothiazide.

Ambulatory follow-up in 3 months revealed office blood pressure of $130 / 70 \mathrm{mmHg}$ and recovery of all symptoms including exertional dyspnea. Echocardiography showed similar findings of LV hypertrophy (septal wall thickness of $20 \mathrm{~mm}$ ) comparable to the initial findings.

\section{Discussion}

The morphological findings and clinical features of LV hypertrophy may be similar to disorders, such as HCM, amyloidosis, and hypertensive heart disease, so misdiagnosis can frequently occur. Our case demonstrates the presence of severe LV hypertrophy, small pericardial effusion, and late gadolinium enhancement by $\mathrm{CMR}$, so infiltrative disease, such as cardiac amyloidosis, was suspected. However, myocardial biopsy revealed the presence of hypertensive heart disease, which could be effectively treated using a combination of antihypertensive drug therapies.

Clinical presentation of patients with LV hypertrophy may differ between absence of clinical signs upon presentation and life-threatening arrhythmias or symptomatic congestive heart failure. Although the 12-lead ECG may be helpful for the identification of HCM [10], such patients might in some cases present with normal ECG. In addition, patients with amyloidosis may exhibit low total 12-lead QRS voltage despite the presence of LV hypertrophy [11].

To date, several imaging methods are available for the diagnostic workup of patients with myocardial hypertrophy. Echocardiography represents the first-line imaging modality for the evaluation of LV hypertrophy, allowing the assessment of LV wall thickness, end-diastolic and end-systolic volumes, and LV mass. In addition, the assessment of diastolic function, myocardial strain, and strain rate by echocardiography 


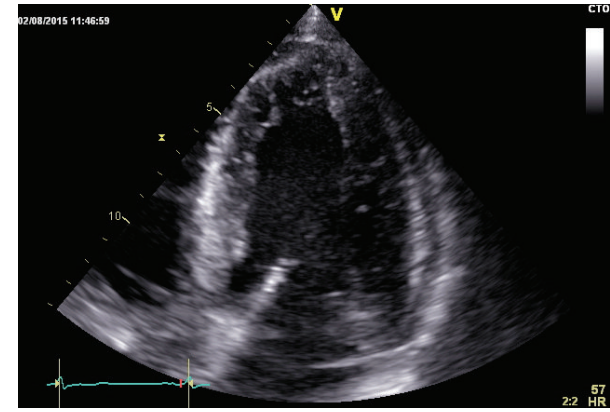

(a)

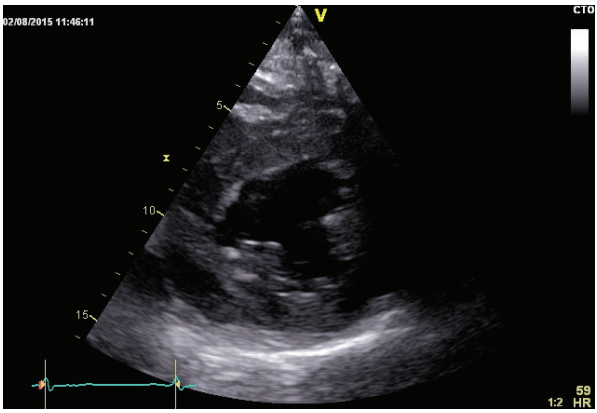

(c)

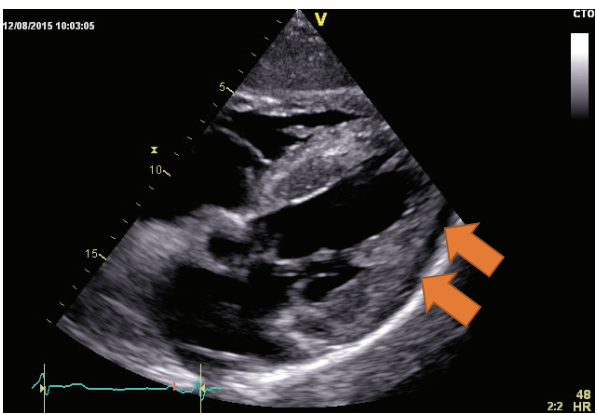

(e)

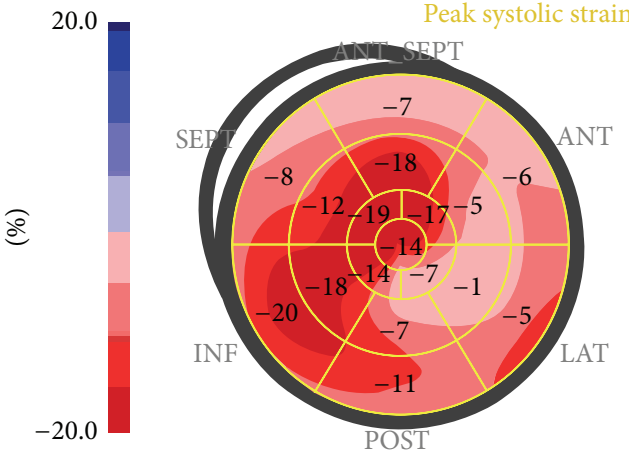

Mean GLS $=-11.1 \%$

(g)

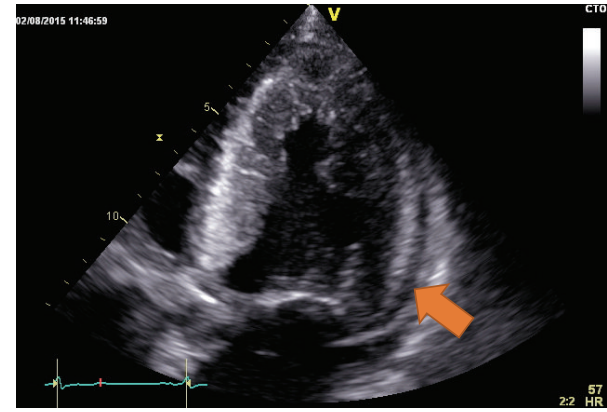

(b)

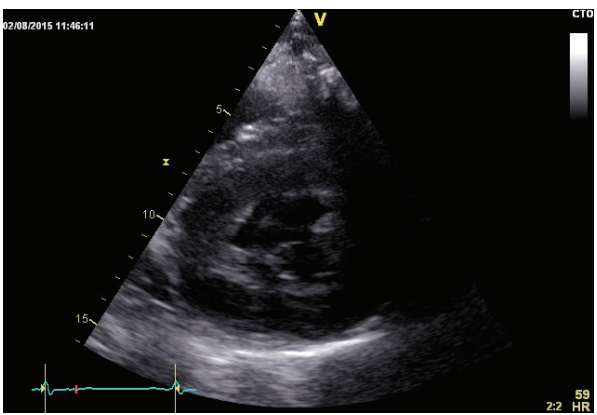

(d)

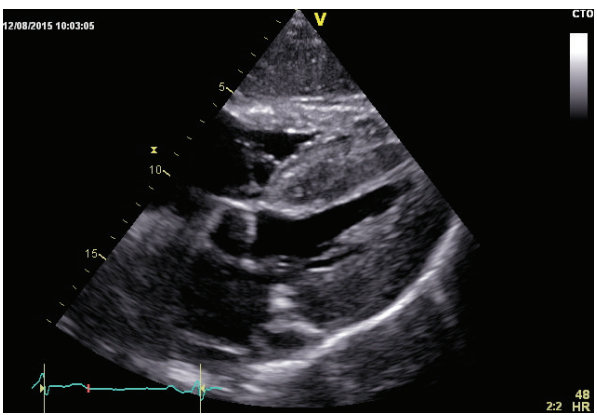

(f)

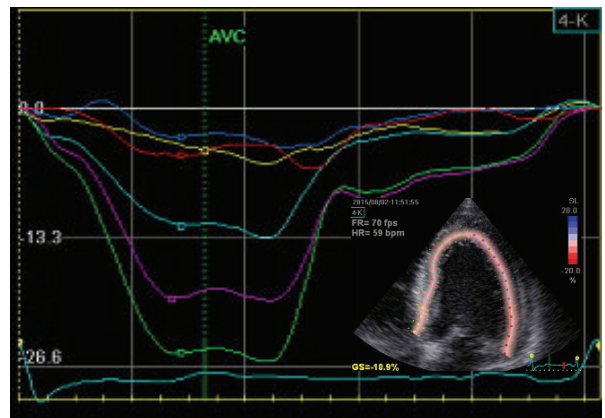

(h)

FIGURE 1: Echocardiographic images revealing severe left ventricular hypertrophy ((a)-(f)). Reduced longitudinal function was noticed in the presence of preserved radial deformation and ejection fraction $((\mathrm{g})-(\mathrm{h}))$. Minimal pericardial effusion was noticed (orange arrows in $(\mathrm{b})$ and (e)). 


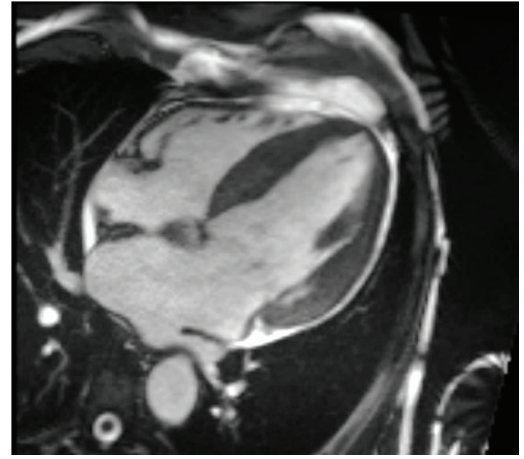

(a)

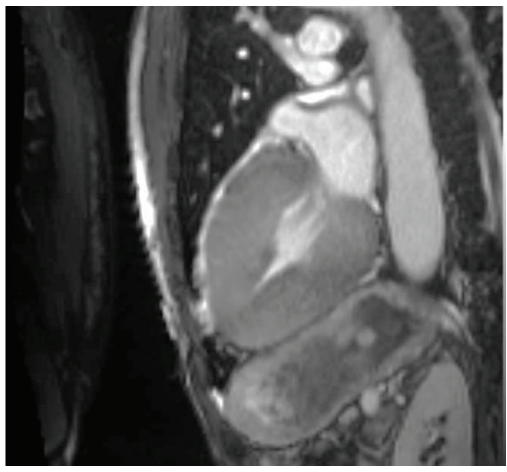

(d)

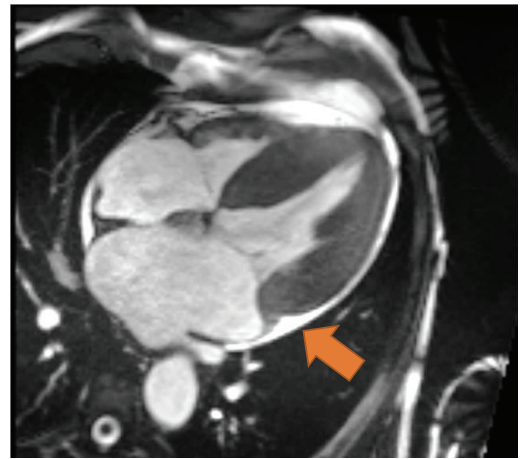

(b)

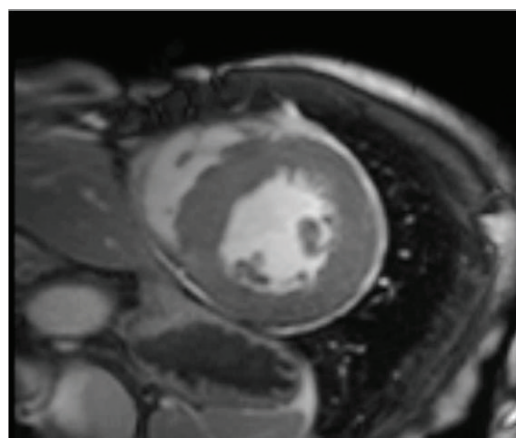

(e)

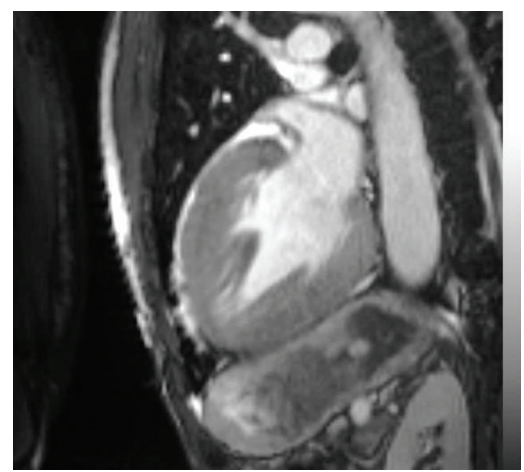

(c)

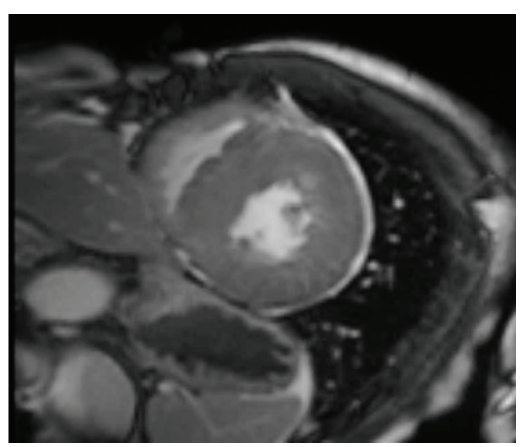

(f)

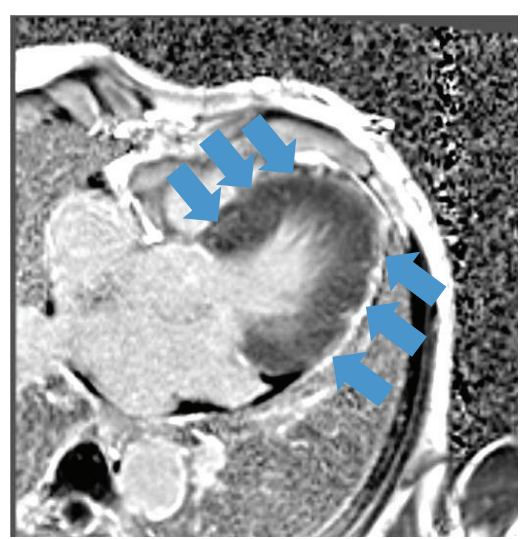

(g)

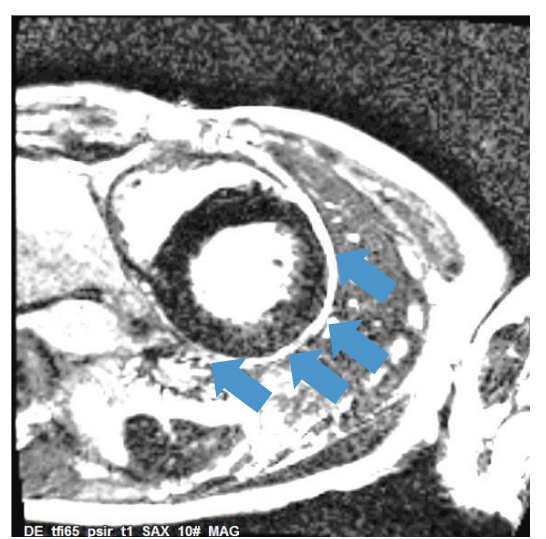

(h)

FIGURE 2: Cardiac magnetic resonance imaging confirmed severe left ventricular hypertrophy and minimal pericardial effusion ((a)-(f), orange arrow in (b)). Late gadolinium enhancement was suspected in corresponding 4-chamber and short axis view images $((\mathrm{g})-(\mathrm{h}))$.

can add incremental value for the differentiation between cardiac amyloidosis and other myocardial disorders causing LV hypertrophy $[12,13]$. Despite its high practicability, however, echocardiography is an operator dependent technique, which is limited by the echogenic windows of the patients.

CMR, on the other hand, exhibits higher spatial resolution than echocardiography, and its versatility allows for the assessment of ventricular thickness and mass, myocardial function (ejection fraction), perfusion, and if required myocardial deformation and viability (scar, fibrosis) by LGE within a single examination and without radiation exposure for the patients [14].
A number of previous studies investigated the ability of CMR to differentiate patients with LV hypertrophy due to HCM, amyloidosis, and hypertensive heart disease. Thus, Sipola et al. [15] suggested that a maximal wall thickness of $\geq 17 \mathrm{~mm}$ could precisely differentiate patients with $\mathrm{D} 175 \mathrm{~N}$ mutation related HCM from those with hypertensive heart disease. However, in our case, this criterion would not be useful, because our patient exhibited a septal wall thickness of over $20 \mathrm{~mm}$ and a maximal wall thickness of $23 \mathrm{~mm}$.

The presence of LGE with hypertensive heart disease, on the other hand, is rated differently in the current literature. Thus, LGE was seen in 14 of 43 patients with HCM but in 


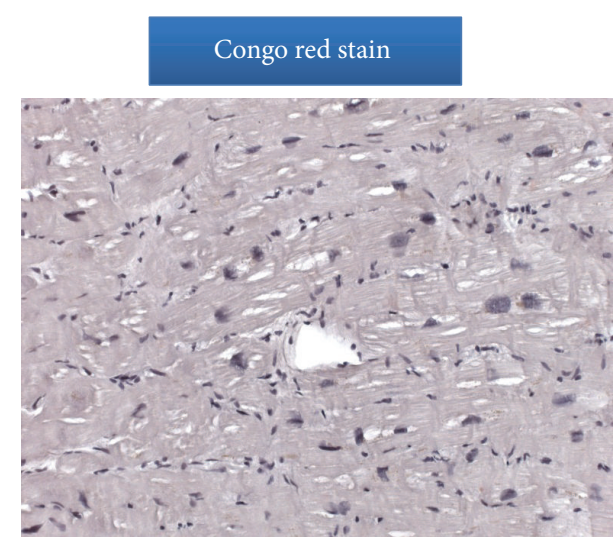

(a)

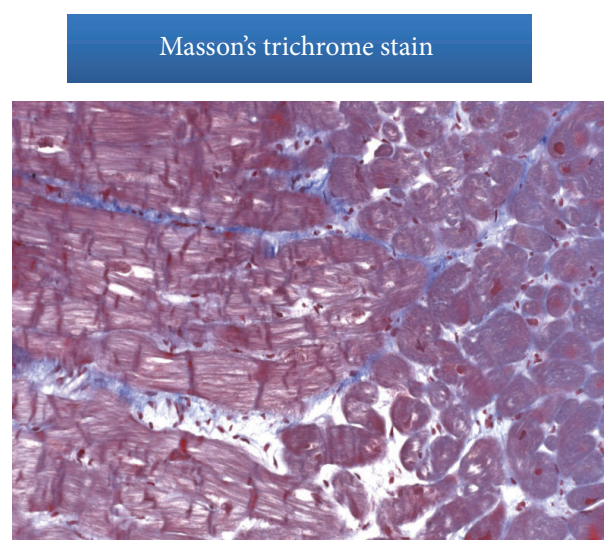

(c)

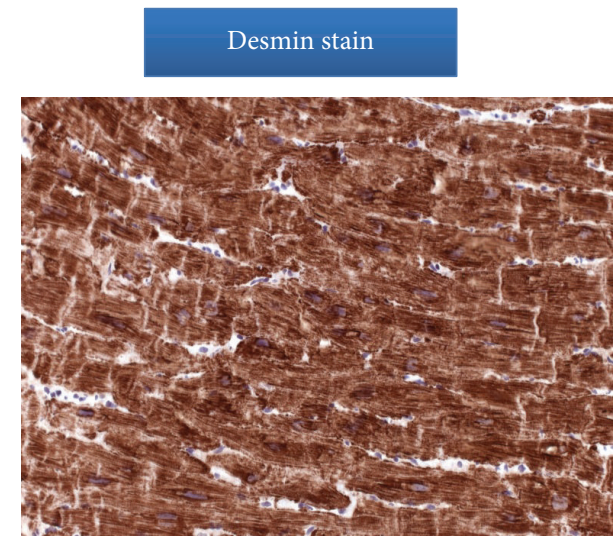

(e)

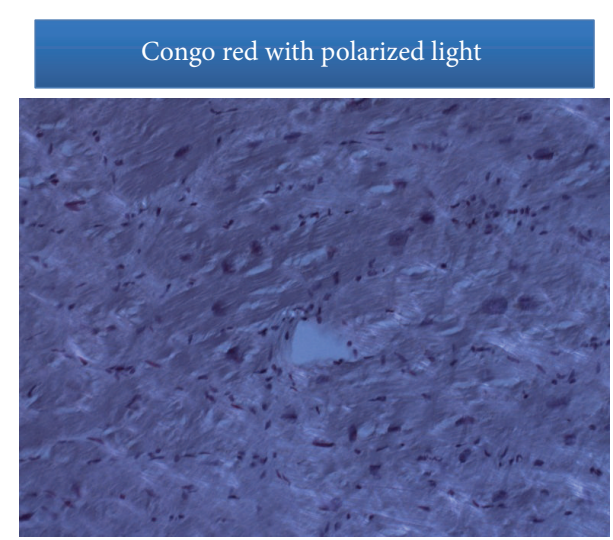

(b)
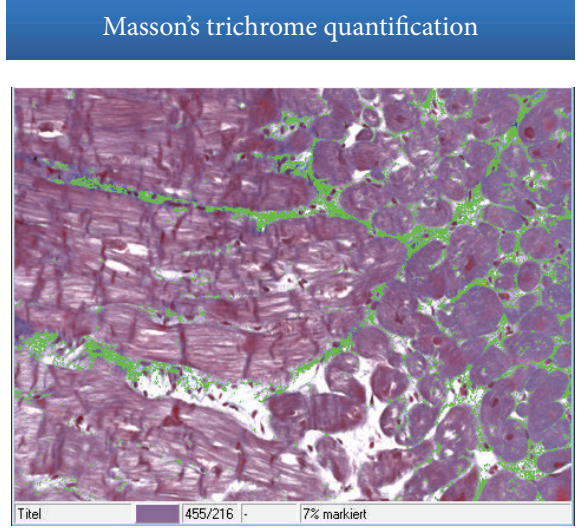

(d)

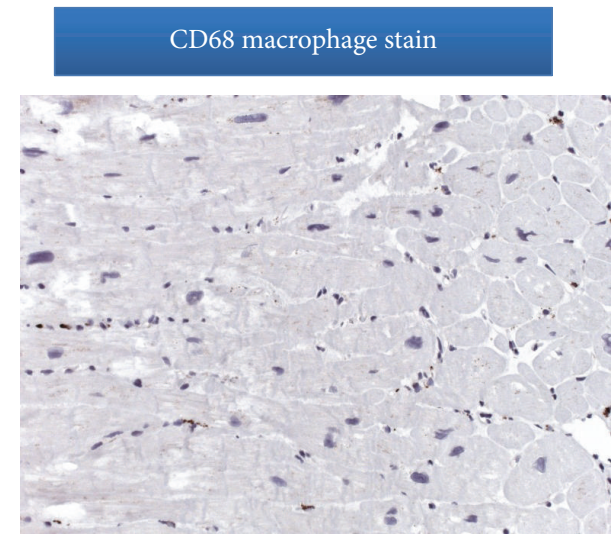

(f)

Figure 3: Congo red stain excluded the presence of amyloid in the heart ((a)-(b)), while Masson's trichrome stain showed diffuse hypertrophy of myocytes and an interstitial fibrosis with the myocardium (blue areas (c), area of fibrosis of $7 \%$ by quantification analysis in (d)), indicating the presence of hypertensive heart disease. In addition, immunohistochemical stain for desmin and CD68 suggested the absence of myocardial texture disorders and macrophage infiltration ((e)-(f)).

none of 39 patients with hypertensive heart disease [16]. In the same line, diffuse LGE was observed in 20 of 30 patients with cardiac amyloidosis, but in none of 16 patients with hypertensive heart disease [17]. In a recent European CMR study, on the other hand, 13 of 26 patients with hypertensive heart disease exhibited nonischemic, patchy LGE, predominantly in septal and inferior-lateral myocardial segments [18]. In the same line, another recent study demonstrated patchy $(n=$ $4)$ and midwall $(n=2)$ nonischemic LGE in 6 of 11 patients with hypertensive heart disease, predominantly in septal and inferior regions [19]. Conversely, in our patient, diffuse LGE was suspected in the 4-chamber view and patchy LGE in the inferior and lateral LV wall. LGE is very common in patients with cardiac amyloidosis and represents interstitial expansion 
from amyloid deposition. Typical findings in cardiac amyloidosis include low $\mathrm{T} 1$ difference between the myocardium and the LV cavity and circumferential subendocardial or transmural LGE [20-23]. In our case, the LGE pattern was not typical for amyloidosis. We therefore decided to proceed with myocardial biopsy.

With hypertensive heart disease, on the other hand, left ventricular hypertrophy results from chronic pressure overload due to systemic hypertension. In the absence of antihypertensive treatment, diastolic dysfunction occurs, which may lead to heart failure symptoms. During later stages of the disease, subendocardial interstitial fibrosis is increasing, compromising myocardial contractility and ultimately causing systolic LV dysfunction [24, 25]. Despite advances in the antihypertensive medications, the clinical burden of hypertensive heart disease is nonnegligible, and in some cases heart failure with preserved ejection function is present due to unchecked arterial hypertension [25]. Due to the increased rate of cardiac events with hypertensive heart disease, the early diagnosis and treatment of this disorder are crucial.

Finally, from a technical point of view, T1 mapping may have further helped in differentiating between amyloidosis, hypertrophic cardiomyopathy, and hypertensive heart disease in our case but was unfortunately not yet implemented with our scanner at the time of the study $[20,26]$.

In conclusion, the diagnostic process with LV hypertrophy requires the integration of clinical evaluation, electrocardiography, echocardiography, and if required CMR, biochemical and serological markers, and endomyocardial biopsy. Although the latest improvements with noninvasive diagnostic techniques have reduced the need for biopsy, in our case, endomyocardial biopsy excluded cardiac amyloidosis, as suspected by CMR, and revealed the presence of hypertensive heart disease.

\section{Competing Interests}

The authors declare that they have no competing interests.

\section{Acknowledgments}

The authors thank Mrs. Monika Forschner, Mrs. Kristina Muend, and Mr. Dimitrios Strauss for the excellent CMR images.

\section{References}

[1] D. Levy, R. J. Garrison, D. D. Savage, W. B. Kannel, and W. P. Castelli, "Prognostic implications of echocardiographically determined left ventricular mass in the Framingham Heart Study," The New England Journal of Medicine, vol. 322, no. 22, pp. 1561-1566, 1990.

[2] M. H. Drazner, "The progression of hypertensive heart disease," Circulation, vol. 123, no. 3, pp. 327-334, 2011.

[3] P. Elliott and W. J. McKenna, "Hypertrophic cardiomyopathy," The Lancet, vol. 363, no. 9424, pp. 1881-1891, 2004.

[4] R. H. Falk, "Cardiac amyloidosis: a treatable disease, often overlooked," Circulation, vol. 124, no. 9, pp. 1079-1085, 2011.
[5] G. Merlini and V. Bellotti, "Molecular mechanisms of amyloidosis," The New England Journal of Medicine, vol. 349, no. 6, pp. 583-596, 2003.

[6] K. M. Harris, P. Spirito, M. S. Maron et al., "Prevalence, clinical profile, and significance of left ventricular remodeling in the end-stage phase of hypertrophic cardiomyopathy," Circulation, vol. 114, no. 3, pp. 216-225, 2006.

[7] S. T. Hardy, L. R. Loehr, K. R. Butler et al., "Reducing the blood pressure-related burden of cardiovascular disease: impact of achievable improvements in blood pressure prevention and control," Journal of the American Heart Association, vol. 4, no. 10, Article ID e002276, 2015.

[8] K. A. L. Mueller, I. I. Mueller, D. Eppler et al., "Clinical and histopathological features of patients with systemic sclerosis undergoing endomyocardial biopsy," PLoS ONE, vol. 10, no. 5, Article ID e0126707, 2015.

[9] J. G. Pickering and D. R. Boughner, "Fibrosis in the transplanted heart and its relation to donor ischemic time. Assessment with polarized light microscopy and digital image analysis," Circulation, vol. 81, no. 3, pp. 949-958, 1990.

[10] W. J. McKenna, P. Spirito, M. Desnos, O. Dubourg, and M. Komajda, "Experience from clinical genetics in hypertrophic cardiomyopathy: proposal for new diagnostic criteria in adult members of affected families," Heart, vol. 77, no. 2, pp. 130-132, 1997.

[11] W. C. Roberts, G. Filardo, J. M. Ko et al., "Comparison of total 12-lead QRS voltage in a variety of cardiac conditions and its usefulness in predicting increased cardiac mass," American Journal of Cardiology, vol. 112, no. 6, pp. 904-909, 2013.

[12] G. Di Bella, F. Minutoli, A. Pingitore et al., "Endocardial and epicardial deformations in cardiac amyloidosis and hypertrophic cardiomyopathy," Circulation Journal, vol. 75, no. 5, pp. 12001208, 2011.

[13] J. P. Sun, W. J. Stewart, X. S. Yang et al., "Differentiation of hypertrophic cardiomyopathy and cardiac amyloidosis from other causes of ventricular wall thickening by two-dimensional strain imaging echocardiography," American Journal of Cardiology, vol. 103, no. 3, pp. 411-415, 2009.

[14] G. Korosoglou, P. M. Humpert, J. Ahrens et al., "Left ventricular diastolic function in type 2 diabetes mellitus is associated with myocardial triglyceride content but not with impaired myocardial perfusion reserve," Journal of Magnetic Resonance Imaging, vol. 35, no. 4, pp. 804-811, 2012.

[15] P. Sipola, J. Magga, M. Husso, P. Jääskeläinen, K. Peuhkurinen, and J. Kuusisto, "Cardiac MRI assessed left ventricular hypertrophy in differentiating hypertensive heart disease from hypertrophic cardiomyopathy attributable to a sarcomeric gene mutation," European Radiology, vol. 21, no. 7, pp. 1383-1389, 2011.

[16] V. O. Puntmann, C. Jahnke, R. Gebker et al., "Usefulness of magnetic resonance imaging to distinguish hypertensive and hypertrophic cardiomyopathy," American Journal of Cardiology, vol. 106, no. 7, pp. 1016-1022, 2010.

[17] A. M. Maceira, J. Joshi, S. K. Prasad et al., "Cardiovascular magnetic resonance in cardiac amyloidosis," Circulation, vol. 111, no. 2, pp. 186-193, 2005.

[18] A. Rudolph, H. Abdel-Aty, S. Bohl et al., "Noninvasive detection of fibrosis applying contrast-enhanced cardiac magnetic resonance in different forms of left ventricular hypertrophy. relation to remodeling," Journal of the American College of Cardiology, vol. 53, no. 3, pp. 284-291, 2009.

[19] M. Takeda, Y. Amano, M. Tachi, H. Tani, K. Mizuno, and S. Kumita, "MRI differentiation of cardiomyopathy showing 
left ventricular hypertrophy and heart failure: differentiation between cardiac amyloidosis, hypertrophic cardiomyopathy, and hypertensive heart disease," Japanese Journal of Radiology, vol. 31, no. 10, pp. 693-700, 2013.

[20] M. Fontana, S. Pica, P. Reant et al., "Prognostic value of late gadolinium enhancement cardiovascular magnetic resonance in cardiac amyloidosis," Circulation, vol. 132, no. 16, pp. 15701579, 2015.

[21] H. Vogelsberg, H. Mahrholdt, C. C. Deluigi et al., "Cardiovascular magnetic resonance in clinically suspected cardiac amyloidosis. noninvasive imaging compared to endomyocardial biopsy," Journal of the American College of Cardiology, vol. 51, no. 10, pp. 1022-1030, 2008.

[22] I. S. Syed, J. F. Glockner, D. Feng et al., "Role of cardiac magnetic resonance imaging in the detection of cardiac amyloidosis," JACC: Cardiovascular Imaging, vol. 3, no. 2, pp. 155-164, 2010.

[23] J. A. White, H. W. Kim, D. Shah et al., "CMR imaging with rapid visual T1 assessment predicts mortality in patients suspected of cardiac amyloidosis," JACC: Cardiovascular Imaging, vol. 7, no. 2, pp. 143-156, 2014.

[24] J. Díez, A. González, B. López, and R. Querejeta, "Mechanisms of disease: pathologic structural remodeling is more than adaptive hypertrophy in hypertensive heart disease," Nature Clinical Practice Cardiovascular Medicine, vol. 2, no. 4, pp. 209216, 2005.

[25] S. V. Raman, "The hypertensive heart. An Integrated Understanding Informed by Imaging," Journal of the American College of Cardiology, vol. 55, no. 2, pp. 91-96, 2010.

[26] R. Hinojar, N. Varma, N. Child et al., "T1 mapping in discrimination of hypertrophic phenotypes: hypertensive heart disease and hypertrophic cardiomyopathy: findings from the international T1 multicenter cardiovascular magnetic resonance study," Circulation: Cardiovascular Imaging, vol. 8, no. 12, Article ID e003285, 2015. 


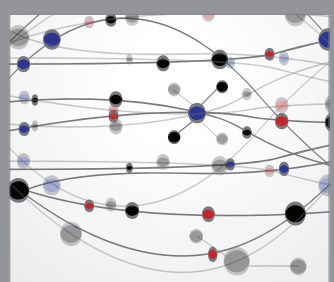

The Scientific World Journal
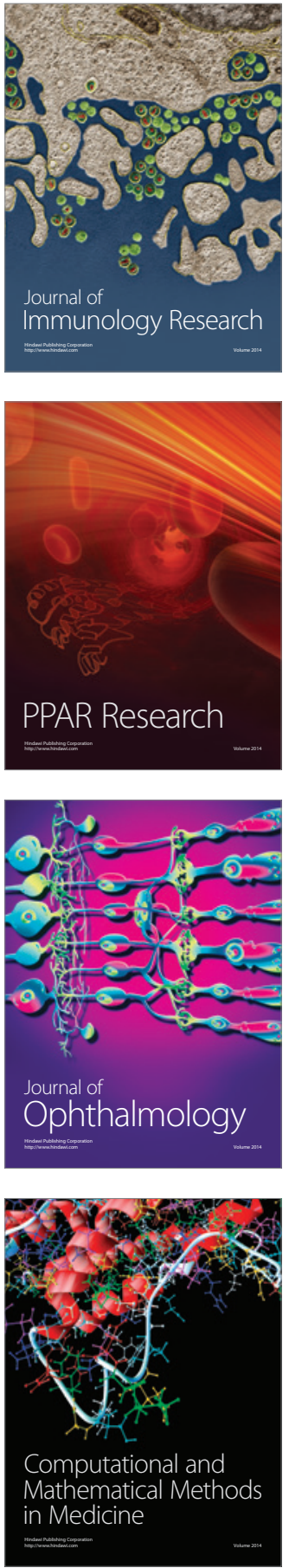

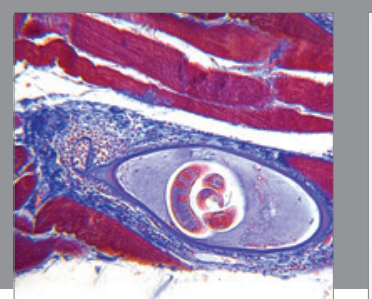

Gastroenterology Research and Practice

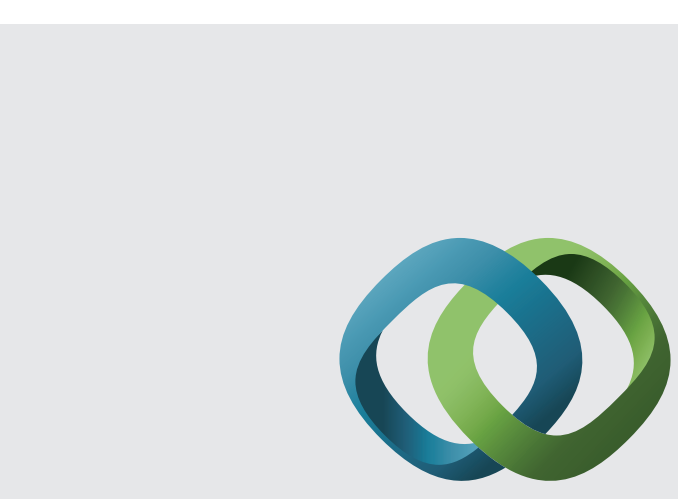

\section{Hindawi}

Submit your manuscripts at

http://www.hindawi.com
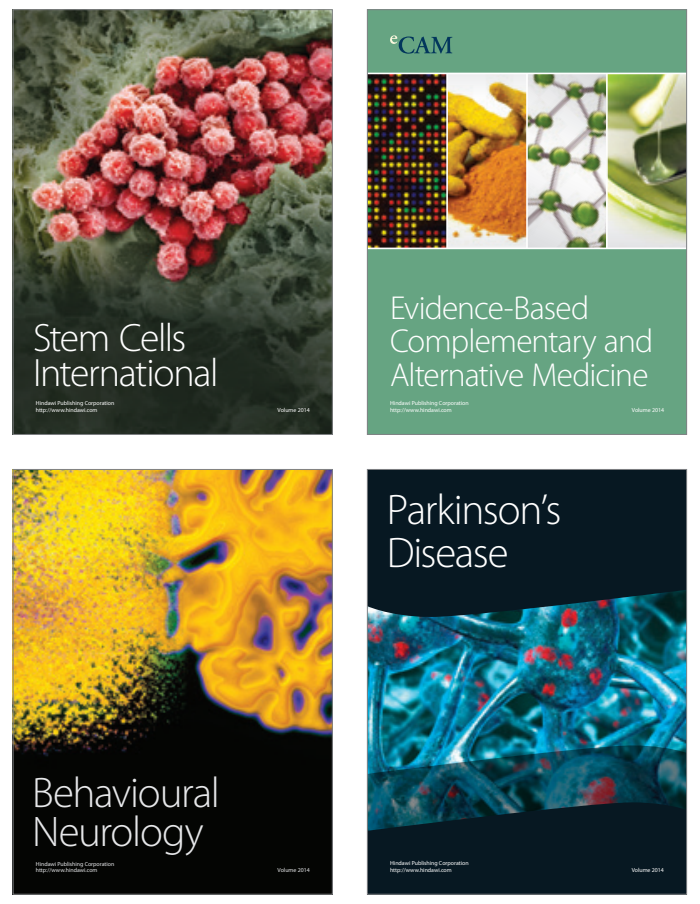
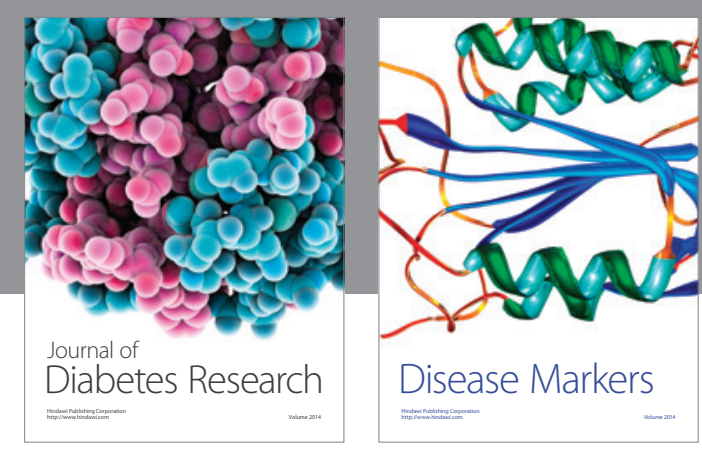

Disease Markers
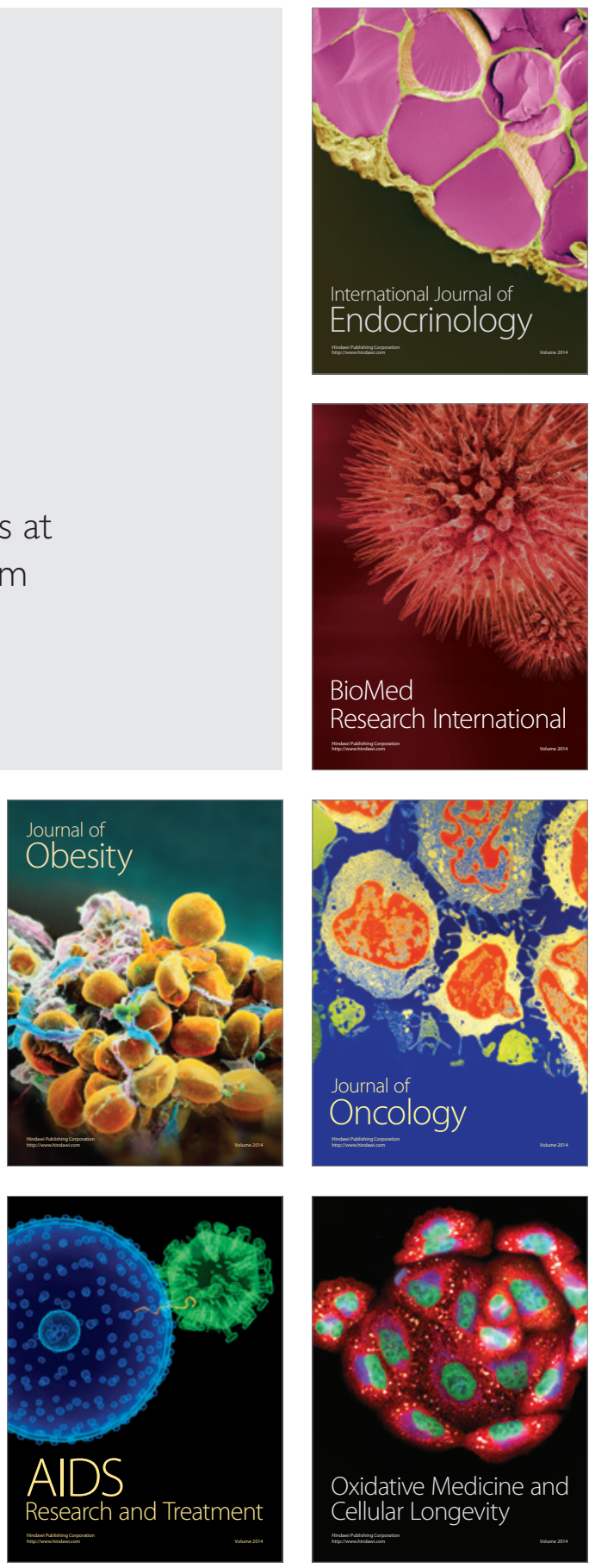